
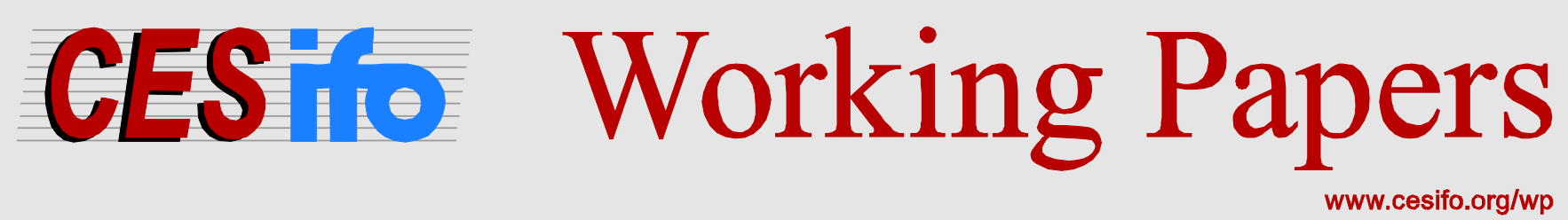

\title{
Negative Campaigning in a Probabilistic Voting Model
}

\author{
Jan K. Brueckner \\ Kangoh Lee
}

CESIFO WORKING PAPER NO. 4233

CATEgory 2: PUBliC CHOICE

MAY 2013

An electronic version of the paper may be downloaded

- from the SSRN website:

- from the RePEc website:

- from the CESifo website:

wWw.SSRN.com

www.RePEc.org

www.CESifo-group.org/wp

\section{CESifo}




\title{
Negative Campaigning in a Probabilistic Voting Model
}

\begin{abstract}
This paper extends the small existing theoretical literature on negative campaigning, building on work by Harrington and Hess (1996). While their analysis explores the determinants of negative campaign spending using a classic spatial voting model, this paper relies instead on a probabilistic voting model, extending the use of this popular model to a new setting. By using two different versions of the model based on different assumptions on the targeting of campaign spending, the paper generates a host of results. However, the main lesson, which emerges in fairly robust fashion regardless of specific assumptions, is that negative campaign spending is higher for the relatively centrist candidate. The more-extreme candidate in the electoral contest devotes, by contrast, comparatively more of her funds to positive campaign spending. This result, which at first appears unrelated to the main findings of Harrington and Hess (1996) and Chakrabarti's (2007) extension of their model, is actually consistent with their results upon deeper examination.
\end{abstract}

JEL-Code: D700.

Keywords: negative campaigning, probabilistic voting.

Jan K. Brueckner

Department of Economics

University of California, Irvine

3151 Social Science Plaza

USA - Irvine, CA 92697

jkbrueck@uci.edu
Kangoh Lee

Department of Economics

San Diego State University

5500 Campanile Drive

USA - San Diego, CA 92182

klee@mail.sdsu.edu

April 2013 


\title{
Negative Campaigning in a Probabilistic Voting Model
}

by

\author{
Jan K. Brueckner and Kangoh Lee*
}

\section{Introduction}

Negative campaigning has become an increasingly common feature of electoral contests in the US, with the recent presidential campaigns of Barack Obama and Mitt Romney offering the latest examples. While political scientists have extensively studied the general effects of campaign spending on electoral outcomes, ${ }^{1}$ the literature focusing specifically on the impact of negative campaign spending (used for critical advertising) is more limited. This literature contains both theoretical and empirical contributions, with economists contributing significantly to the theoretical component.

One strand of the theoretical literature analyzes negative campaigning using a spatial median-voter model in the tradition of Hotelling (1929) and Downs (1957). Under this approach, initiated by Harrington and Hess (1996) and extended by Chakrabarti (2007), a candidate's negative campaign spending serves to push the perceived policy position of the opponent toward a more-extreme location, shifting votes toward the candidate. "Positive" campaign spending by the opponent attempts to offset this effect, preventing the loss of votes. The purpose of the present paper is to extend this spatial approach by using a probabilistic voting model in place of the classic median-voter framework. This type of model (see, for example, Lindbeck and Weibull (1987), Enelow and Hinich (1989), Grossman and Helpman (1996), Persson and Tabellini (Ch. 3, 2000)) offers a more-realistic picture of the voting process, overturning some unrealistic implications of the median-voter model.

In that model, a candidate has an incentive to chose a position just on the median side

of her opponent's position, ${ }^{2}$ thereby capturing all the votes on that side of the opponent and winning the election. Under the probabilistic voting model, however, individual vote outcomes are stochastic due to the presence of a random, idiosyncratic "valence" effect (which shifts a 
candidate's attractiveness) along with other shocks that affect all voters in common. Since a voter's preferred policy position is then not a perfect indicator of how he will vote, locating adjacent to the opponent is not the right strategy for a candidate. Under the version of the model used in this paper, a candidate's best strategy would be to locate at the median of the voters' preferred positions regardless of the opponent's location, avoiding a potential vote loss from a more-extreme position. With the other candidate making the same choice, the equilibrium candidate positions would then have the same common median location as in the median-voter model. But the mechanics of the models are different, and once negative and positive campaign spending are introduced, an entirely new analysis is needed. Thus, an exploration of negative campaigning in a probabilistic voting model is a worthwhile undertaking that can advance our understanding of the behavior of political candidates.

The analysis considers two different models of negative campaigning distinguished by the roles of positive spending. In the first model, which follows the setup of Harrington and Hess (1996, hereafter HH), both positive and negative spending shift the candidates' positions, as perceived by the voters. More specifically, a candidate's positive spending moves her perceived position toward the median while her negative spending moves the perceived position of the opponent away from the median. A candidate's positive spending thus makes her look more centrist while negative spending makes her opponent look more extreme. In the second model, negative spending plays the same role as in the first model, but positive spending affects the perceived valences of the candidates, shifting the distribution of the random valence variable that captures the relative attractiveness of the two candidates. Charkabarti's (2007) modification of the $\mathrm{HH}$ model is in a similar spirit, but the details are different.

In both models, the stated policy position of a candidate, perceptions of which are shifted by negative and positive spending, is also a choice variable, in contrast to the exogeneity of stated positions in the models of $\mathrm{HH}$ and Chakrabarti. Each candidate has an underlying belief or ideology, and the candidates are not purely office-driven, suffering utility losses when their stated positions diverge from these ideologies. The candidates are also constrained by fixed total campaign budgets, which are split between negative and positive spending. Taking her budget constraint into account, a candidate then has two choice variables: stated position and 
the level of negative campaign spending. These variables are set in Nash fashion to maximize a utility function that equals the probability of winning times the benefit of holding office minus the loss due to divergence from ideology. In addition to analyzing the full choice problem, the discussion also considers the simpler case where the candidates' stated positions are fixed rather than chosen, as in the previous papers. However, along with the application of the probabilistic voting model, the endogeneity of stated positions is a significant extension of the previous frameworks.

The main goal of the analysis is to draw connections between the levels of negative campaign spending and the parameters of the model. The budget levels are important parameters, and their impacts are mostly positive, as expected. The analysis also shows the effects of parameters that capture the disutility of divergence from ideology and the productivity of negative campaign spending, with the latter effect naturally positive. The most important results, however, concern the effect of a candidate's relative centrism on her level of negative spending. When the stated policy positions are choice variables, centrism is captured by ideology, but when the stated positions are fixed, centrism is captured by the positions themselves. The main result of the paper is that, when centrism is asymmetric, the more-centrist candidate has the higher level of negative campaign spending.

This result, which holds regardless of whether stated positions are chosen or fixed, may at first seem counterintuitive: a centrist candidate might seem to have little incentive to make her less-centrist opponent seem even more extreme through negative spending. The explanation, however, involves the central feature of the probabilistic voting model, as developed in the current paper. In particular, a candidate's gain from becoming marginally more centrist diminishes as her perceived position moves toward the median. Conversely, the candidate's gain from her opponent becoming marginally more extreme (which equals the opponent's loss from this movement) becomes larger as the opponent's perceived position moves away from the median. Therefore, a centrist candidate has a small marginal gain from extra positive spending and relatively large marginal gain from extra negative spending, causing spending to shift in the negative direction until (as a result of diminishing returns) these gains are equated. Harrington and Hess (1996) show that the candidate with the higher valence (which is 
nonstochastic and common across voters) spends relatively less on negative campaigning than her lower-valence opponent. Although this conclusion at first appears unconnected to the result described above, the two findings are roughly consistent, as follows. Since the two candidates appeal equally to the decisive voter in the HH model, the higher-valence candidate's position is farther from the decisive voter's preferred position than is the opponent's. The higher-valence candidate is thus the more-extreme candidate, and her lower negative spending therefore matches the current result. However, given the probabilistic nature of the current model and the assumption that voter utility is linear rather than strictly convex in the position differences, the mechanisms underlying the results in the two papers are very different.

Chakrabarti (2007) extends HH by assuming two types of negative spending: ideological spending that shifts the opponent's policy position away from the median and valence spending that reduces the opponent's valence index. Chakrabarti's model is thus similar to the second model in the current paper, except that valence spending has a negative rather than positive effect (a difference that is inessential). His main result is that the higher-valence candidate (who is less centrist from above) engages in valence-shifting spending, which corresponds to positive spending in the current model. The lower-valence, more-centrist candidate focuses on negative, position-shifting spending. Therefore, Chakrabarti's results match the current findings, although the underlying mechanisms are different.

In other work, Skaperdas and Grofman (1995) and Polborn and Yi (2006) consider nonspatial models of negative campaigning. In Skaperdas and Grofman's model, positive campaigning attracts undecided voters to a candidate while negative campaigning turns the opponent's supporters into undecided voters. They show that the front-runner devotes more resources to positive campaigning, while the lagging opponent spends more on negative campaigning. In Polborn and Yi's model, the only way unfavorable factual information about a candidate can be transmitted is through the opponent's negative campaigning. Candidates must choose whether to spend their budget in this fashion to reduce support for the opponent or whether to tout their own virtues via positive spending. Their results show that a candidate engages in positive campaigning if she can reveal positive information about herself or if the opponent does not have significant weaknesses. ${ }^{3}$ 
A significant empirical literature focuses on negative campaigning, with a useful survey provided by Lau and Rovner (2009). Some papers ask whether negative campaigning "demobilizes" the electorate, disenchanting voters and thus reducing turnout. This view was advanced by Ansolabehere, Iyengar, Simon and Valentino (1994) but questioned by Wattenberg and Brians (1999) and others. The studies in this literature most directly related to the current paper measure the determinants of negative campaigning. These papers include Damore (2002), Haynes and Rhine (1998), Kahn (1993), Lau and Pomper (2001), Proctor,

Peterson and Djupe (2005), and Schenck-Hamlin and Haase (1994), among others. Relying on various types data on campaign negativism, the studies focus on potential causal factors such as the support difference between candidates, presence of an incumbent, party affiliation, gender, and proximity to the election date. However, none of their findings are directly relevant to the current paper's main prediction regarding the link between centrism and negativity.

The plan of the paper is as follows. Section 2 presents the probabilistic voting model, developing it using the perceived policy positions of the candidates without specifying how they are determined. Sections 3 and 4 present the two versions of the model, which make different assumptions about the links between perceived positions and the levels of campaign spending. Section 5 offers conclusions.

\section{A Model of Probabilistic Voting}

The model has a continuum of voters with unit mass and two candidates, 1 and 2. Voters differ in their ideology, $x$, which is distributed over the support $[0,1]$ according to the cumulative distribution function $F(x)$, with $f(x)=F^{\prime}(x)$. For notational simplicity, $f(x)$ is assumed symmetric and unimodal. The mean and the median of $x$ are thus the same and equal to $1 / 2$, with $F(1 / 2)=1 / 2$. Let $Z_{i}$ denote the policy position of candidate $i$, as perceived by voters, and assume that

$$
Z_{1}<1 / 2<Z_{2} .
$$

Voters thus perceive candidate 1 as liberal and candidate 2 as conservative. Further foundations for (1) will be provided below. For later use, observe that the inequalities in (1), along with 
$F(1 / 2)=1 / 2$, imply

$$
1-2 F\left(Z_{1}\right)>0, \quad 2 F\left(Z_{2}\right)-1>0
$$

As will be discussed in the subsequent sections, $Z_{i}$ depends on the stated policy position of candidate $i$ and the campaign spending of both candidates. This section, however, derives the probability of a candidate winning the election regardless of how $Z_{i}$ is determined, a probability that plays a key role in the analysis.

Voter $x$ 's utility depends linearly on the distance between his ideology $x$ and a candidate's perceived position $Z_{i}$. A voter's utility also depends on his idiosyncratic preference for candidate 1 and on a random shock. The utility for voter $x$ is written as $-\left|x-Z_{1}\right|+v_{x}+s$ when choosing candidate 1 , and $-\left|x-Z_{2}\right|$ when choosing candidate 2 . The variable $s$ denotes a random political shock, such as a terrorist attack or a serious economic downturn, that shifts all voters' perceptions of candidate 1 relative to candidate 2 in the same fashion. This shock variable is assumed to be uniformly distributed on the interval $[-\alpha, \alpha]$, where $\alpha>0$. The variable $v_{x}$ captures voter $x$ 's bias toward candidate 1 , or a measure of candidate 1's valence relative to candidate 2's valence, as perceived by this voter. This relative-valence measure is a random variable for each voter and is distributed uniformly on $[-\beta, \beta]$ for all $x$, where $\beta>0$. The valence measure thus has a common distribution centered at zero for all voters, implying that the measure is uncorrelated with a voter's ideology $x$. While this assumption could be relaxed by allowing $\beta$ to be a function of $x$ (so that the variance of $v_{x}$ is voter-specific), altering the assumption that the distribution is centered at zero for all voters would make the analysis intractable. This type of probabilistic voting model has been used in the political-economy literature (for example, Lindbeck and Weibull (1987), Enelow and Hinich (1989), Grossman and Helpman (1996), Persson and Tabellini (Ch. 3, 2000)).

To calculate the probability that candidate $i$ wins, consider voters' decisions. Voter $x$ votes for candidate 1 if

$$
-\left|x-Z_{1}\right|+v_{x}+s \geq-\left|x-Z_{2}\right|,
$$


where voter is assumed to vote for candidate 1 if indifferent between the two candidates. The inequality in (3) depends on the voter's identity $x$, and three cases are considered. For $x<Z_{1}$, (3) reduces to $-\left(Z_{1}-x\right)+v_{x}+s>-\left(Z_{2}-x\right)$ and hence $v_{x}>Z_{1}-Z_{2}-s$, so that the probability voter $x$ votes for candidate 1 is

$$
\int_{Z_{1}-Z_{2}-\phi}^{\beta} \frac{1}{2 \beta} d v_{x}=\frac{1}{2}+\frac{1}{2 \beta}\left(Z_{2}-Z_{1}+s\right)
$$

where $1 / 2 \beta$ is the density function of $v_{x}$. For $x \in\left[Z_{1}, Z_{2}\right]$, (3) becomes $-\left(x-Z_{1}\right)+v_{x}+s>$ $-\left(Z_{2}-x\right)$ or $v_{x} \geq 2 x-Z_{1}-Z_{2}-s$, so that the probability voter $x$ votes for candidate 1 is

$$
\int_{2 x-Z_{1}-Z_{2}-\phi}^{\beta} \frac{1}{2 \beta} d v_{x}=\frac{1}{2}+\frac{1}{2 \beta}\left(Z_{1}+Z_{2}-2 x+s\right) .
$$

In an analogous manner, for $x \geq Z_{2}$, the probability that voter $x$ votes for candidate 1 is

$$
\int_{Z_{2}-Z_{1}-s}^{\beta} \frac{1}{2 \beta} d v_{x}=\frac{1}{2}+\frac{1}{2 \beta}\left(Z_{1}-Z_{2}+s\right)
$$

The expected number of votes received by candidate 1 is computed by weighting the probabilities in (4)-(6) by the numbers of voters for each case, which equal $F\left(Z_{1}\right), F\left(Z_{2}\right)-$ $F\left(Z_{1}\right)$, and $1-F\left(Z_{2}\right)$, respectively, and summing. The result is

$$
\frac{1}{2}+\frac{s}{2 \beta}+\frac{1}{2 \beta}\left[-2 Z_{1} F\left(Z_{1}\right)+2 Z_{2} F\left(Z_{2}\right)+Z_{1}-Z_{2}\right]-\int_{Z_{1}}^{Z_{2}} \frac{x}{\beta} f(x) d x .
$$

Integrating the last term in (7) by parts, ${ }^{4}$ substituting the result, and simplifying, (7) reduces to

$$
\frac{1}{2}+\frac{s}{2 \beta}+\frac{1}{2 \beta}\left[Z_{1}-Z_{2}+2 \int_{Z_{1}}^{Z_{2}} F(x) d x\right] .
$$

The probability that candidate 1 wins the election is the probability that his number of supporters, given by (8), exceeds $1 / 2$, or the probability that $s>Z_{1}-Z_{2}+2 \int_{Z_{1}}^{Z_{2}} F(x) d x \equiv k$. 
This probability is $\int_{k}^{\alpha} \frac{1}{2 \alpha} d s$, where $1 / 2 \alpha$ is the density of $s$. Evaluating this integral, candidate 1 's probability of winning is then

$$
\pi_{1} \equiv \frac{1}{2}+\frac{1}{2 \alpha}\left[Z_{1}-Z_{2}+2 \int_{Z_{1}}^{Z_{2}} F(x) d x\right]
$$

Candidate 2's probability of winning is $\pi_{2}=1-\pi_{1}$.

Intuitively, $\partial \pi_{1} / \partial Z_{1}=1-2 F\left(Z_{1}\right)>0$ by $(2)$, so that the probability of candidate 1 winning increases as her position moves closer to the median. Analogously, $\partial \pi_{1} / \partial Z_{2}=2 F\left(Z_{2}\right)-1>0$. Obviously, the effects of $Z_{1}$ and $Z_{2}$ on $\pi_{2}$ are the opposite of those on $\pi_{1}$. Therefore, when $Z$ 's are chosen directly, $Z_{1}$ and $Z_{2}$ in the Nash equilibrium are both set at the median preferred policy position of voters, as noted in the introduction.

Note that, underlying these choices, is the following sequence of events. First, the candidates choose their $Z$ 's, with an understanding of the random nature of the voting process. Then, the random process generates realized values of the valence variables $v_{x}$ for all voters and a value of the common random shock $s$. Votes are then cast, and a winner is determined.

\section{The Choice of Position-Shifting Campaign Spending}

\subsection{The model and first-order conditions}

Let $X_{i}$ denote the stated policy position of candidate $i$. Without loss of generality, assume $0<X_{1}<X_{2}<1$, so that candidate 1 is again liberal and candidate 2 conservative. Voters imperfectly observe $X_{i}$, with campaign spending influencing the perceived positions of the candidates. Letting $P_{i}$ and $N_{i}$ denote the positive and negative campaign spending of candidate $i$, the voter-perceived positions of the candidates are given by

$$
\begin{aligned}
& Z_{1}=X_{1}+h\left(P_{1}\right)-\lambda h\left(N_{2}\right) \\
& Z_{2}=X_{2}-h\left(P_{2}\right)+\lambda h\left(N_{1}\right),
\end{aligned}
$$

where $h^{\prime}>0, h^{\prime \prime}<0, h(0)=0$ and $\lambda>0 .{ }^{5}$ Thus, as in Harrington and Hess (1996), a candidate's negative spending shifts her opponent's perceived position to a more-extreme 
location, leftward for candidate 1 and rightward for candidate 2. Positive spending has the reverse effect, tending to offset the distortionary effect of negative spending by moving a candidate's perceived position back toward the center. The productivity parameter $\lambda$ indicates the potency of negative campaigning relative to positive campaigning. When $\lambda>1$, for example, positive spending is unable to reverse the effect of the same amount of negative spending. 6

Candidate $i$ has a campaign budget, $E_{i}$, to be allocated between $N_{i}$ and $P_{i}$. To avoid uninteresting and unrealistic cases, assume that

$$
X_{1}+h\left(E_{1}\right)-\lambda h(0)=X_{1}+h\left(E_{1}\right)<1 / 2<X_{2}-h\left(E_{2}\right)=X_{2}-h\left(E_{2}\right)+\lambda h(0) .
$$

The inequalities in (12) imply that $Z_{1}<1 / 2<Z_{2}$, as in (1), and thus yield (2) for all combinations of $\left(N_{1}, P_{1}, N_{2}, P_{2}\right)$. Voters then perceive candidate 1 to be liberal, with $Z_{1}<1 / 2$, and candidate 2 to be conservative, with $Z_{2}>1 / 2$, regardless of the levels of campaign spending.

Candidate $i$ 's utility reflects the benefit from winning the election, given by the win probability $\pi_{i}$ times a value $R$, which captures the benefits or "rent" from holding office (assumed for simplicity to be the same for both candidates). Subtracted from this gain is the candidate's loss from stating a policy position $X_{i}$ that diverges from her own ideology. This loss is represented as $\gamma\left(X_{i}-B_{i}\right)^{2}$, where $B_{i}$ gives $i$ 's ideology (or "beliefs") and $\gamma>0$ captures the sensitivity of utility to divergence from ideology. Candidates $i$ 's utility is thus written $\pi R-\gamma\left(X_{i}-B_{i}\right)^{2}$, and after substituting (10) and (11) into the $\pi_{1}$ expression from (9), candidate 1's utility is written as

$$
\begin{aligned}
& U_{1}= \\
& \begin{aligned}
R\left(\frac{1}{2}+\frac{1}{2 \alpha}\left[X_{1}+h\left(P_{1}\right)-\lambda h\left(N_{2}\right)\right.\right. & \left.\left.-X_{2}+h\left(P_{2}\right)-\lambda h\left(N_{1}\right)+2 \int_{X_{1}+h\left(P_{1}\right)-\lambda h\left(N_{2}\right)}^{X_{2}-h\left(P_{2}\right)+\lambda h\left(N_{1}\right)} F(x) d x\right]\right) \\
& -\gamma\left(X_{1}-B_{1}\right)^{2} .
\end{aligned}
\end{aligned}
$$


Candidate 2's utility is given by (13) with the first plus sign replaced by a minus sign and with $\gamma\left(X_{2}-B_{2}\right)^{2}$ replacing the last expression.

Candidate $i$ chooses her stated policy position $X_{i}$ and campaign spending levels, $P_{i}$ and $N_{i}$, to maximize utility subject to the budget constraint $P_{i}=E_{i}-N_{i}$. Letting superscripts denote partial derivatives, candidate 1's first-order conditions are

$$
\begin{aligned}
& U_{1}^{X_{1}} \simeq\left(1-2 F_{1}\right)-\mu\left(X_{1}-B_{1}\right)=0 \\
& U_{1}^{N_{1}} \simeq\left(2 F_{2}-1\right) \lambda h^{\prime}\left(N_{1}\right)-\left(1-2 F_{1}\right) h^{\prime}\left(P_{1}\right)=0
\end{aligned}
$$

where $F_{i} \equiv F\left(Z_{i}\right), \simeq$ means "proportional to," and $\mu=4 \alpha \gamma / R$. Analogously, the conditions for candidate 2 are

$$
\begin{aligned}
& U_{2}^{X_{2}} \simeq-\left(2 F_{2}-1\right)-\mu\left(X_{2}-B_{2}\right)=0 \\
& U_{2}^{N_{2}} \simeq\left(1-2 F_{1}\right) \lambda h^{\prime}\left(N_{2}\right)-\left(2 F_{2}-1\right) h^{\prime}\left(P_{2}\right)=0 .
\end{aligned}
$$

It is assumed that these conditions generate interior solutions for all the choice variables.

The interpretations of the conditions are straightforward. In choosing her policy position $X_{1}$, candidate 1 balances the benefit of increasing her winning probability (first term in (14)) and the cost of deviating from her ideology (second term). An increase in negative spending $N_{1}$ moves candidate 2's perceived position away from the center (first term in (15)), increasing the probability of candidate 1 winning. But the associated decrease in positive spending $P_{1}$ moves candidate 1's perceived position away from the center (second term in (15)), decreasing the probability of winning. These two effects are balanced in choosing $N_{1}$. Analogous interpretations apply to candidate 2 .

The second-order conditions for candidate 1 include

$$
\begin{gathered}
U_{1}^{X_{1} X_{1}} \simeq-2 f_{1}-\mu<0 \\
U_{1}^{N_{1} N_{1}} \simeq 2 f_{2}\left(\lambda h^{\prime}\left(N_{1}\right)\right)^{2}+\left[2 F_{2}-1\right] \lambda h^{\prime \prime}\left(N_{1}\right)-2 f_{1}\left(h^{\prime}\left(P_{1}\right)\right)^{2}+\left[1-2 F_{1}\right] h^{\prime \prime}\left(P_{1}\right)<0
\end{gathered}
$$


where $f_{i} \equiv f\left(Z_{i}\right)$. While condition (18) holds, (19) is not necessarily satisfied since the term $2 f_{2}\left(\lambda h^{\prime}\left(N_{1}\right)\right)^{2}$ is positive while all other terms are negative. However, since the first and third terms in (19) cancel at a symmetric equilibrium (where $f_{2}=f_{1}$ ), leaving only negative terms, the condition is satisfied at such an equilibrium. It can be shown, though, that (19) holds in general if the function $h$ satisfies $h^{\prime} / h \leq-h^{\prime \prime} / h^{\prime} .7$ If $h$ is a power function, this condition requires that the positive exponent is no larger than $1 / 2$. The additional second-order condition, positivity of the Hessian determinant of $U_{1}$, is not ensured (even under symmetry)

and must be assumed (as seen in the appendix, the $U_{1}^{X_{1} N_{1}}$ term in the determinant is always positive). Note that for analysis of the fixed- $X$ case, which is carried out in the next section, the second-order condition consists only of (19). For this case, the condition is satisfied at a symmetric equilibrium and is satisfied globally if the above sufficient condition holds.

\subsection{Analysis with fixed $X$ 's}

To focus on the choice of campaign spending in a simple manner, this section assumes that the policy positions $X_{1}$ and $X_{2}$ are fixed. Only the first-order conditions (15) and (17) are then relevant, and combining these conditions and eliminating the $P$ 's yields

$$
\frac{1-2 F_{1}}{2 F_{2}-1}=\frac{\lambda h^{\prime}\left(N_{1}\right)}{h^{\prime}\left(E_{1}-N_{1}\right)}=\frac{h^{\prime}\left(E_{2}-N_{2}\right)}{\lambda h^{\prime}\left(N_{2}\right)} .
$$

Suppose that the candidates' campaign budgets are equal (with $E_{1}=E_{2}=E$ ) and that the $X^{\prime}$ 's are symmetric around the median policy position, with $X_{1}=1-X_{2}$. Then (20) is satisfied by a symmetric outcome, where $N_{1}=N_{2}=N$, although asymmetric equilibria cannot be ruled out despite the symmetry of the $E$ 's and $X$ 's. In this symmetric equilibrium, $Z_{1}=1-Z_{2}$ holds from (10) and (11), so that the first ratio in (20) equals 1 , with the second and third ratios equalling 1 as well. Therefore, the $N$ solution is given by

$$
\frac{\lambda h^{\prime}(N)}{h^{\prime}(E-N)}=1
$$

Inspection of $(21)$ shows that $N>(<) E / 2$ holds as $\lambda>(<) 1$, and more generally, the inequality $\partial N / \partial \lambda>0$ is easily established by differentiating (21). In addition, the common $N$ is independent of the $X^{\prime}$ 's, and differentiation of (21) shows that $\partial N / \partial E>0 .{ }^{8}$ 
The analysis now turns to a comparative-static analysis of the fixed- $X$ case. To compute the comparative-static derivatives, the first-order conditions for the $N^{\prime}$ s ((15) and (17)) are totally differentiated. Since the second-order condition (19) and the corresponding condition for candidate 2 are satisfied at a symmetric equilibrium, comparative-static effects at such an equilibrium are thus the focus, with parameter changes then shifting the outcome to a nearby asymmetric equilibrium. However, if the second-order conditions hold globally, as would be the case if $h^{\prime} / h<-h^{\prime \prime} / h^{\prime}$, then the results hold globally as well.

The positive effect of $\lambda$ on negative spending in a symmetric equilibrium was just established in the discussion of (21). Additional results, which are established in the appendix, show the effect of campaign budgets on the spending levels:

$$
\frac{\partial N_{i}}{\partial E_{i}}>0, \quad \frac{\partial N_{i}}{\partial E_{j}}<0, \quad i, j=1,2, \quad j \neq i
$$

Therefore, negative spending is increasing in the size of candidate's own budget and decreasing in the size of her opponent's budget. Appraising the effect of $E_{i}$ on own positive spending requires knowledge of the magnitude of the derivative $\partial N_{i} / \partial E_{i}$ relative to 1 , given that $\partial P_{i} / \partial E_{i}=\partial\left(E_{i}-N_{i}\right) / \partial E_{i}=1-\partial N_{i} / \partial E_{i}$. While this relationship is ambiguous, making $\partial P_{i} / \partial E_{i}$ ambiguous, an increase in the opponent's budget raises positive spending since $\partial P_{i} / \partial E_{j}=-\partial N_{i} / \partial E_{j}>0$.

To use (22) to make a simpler statement, suppose $X_{1}=1-X_{2}$ and $E_{1}=E_{2}=E$, and let the symmetric equilibrium that exists under these conditions be the starting point. Then, suppose $E_{1}$ rises and $E_{2}$ falls, so that $E_{1}>E_{2}$. Using (22), the rise in $E_{1}$ and drop in $E_{2}$ cause $N_{1}$ to rise and $N_{2}$ to fall, so that $N_{1}>N_{2} \cdot{ }^{9}$ The following natural conclusion can then be stated:

Proposition 1. In the vicinity of a symmetric equilibrium where stated positions are fixed, the candidate with the larger budget has the higher level of negative campaign spending.

The exogenous stated positions of the candidates also affect spending choices. The ap- 
pendix establishes the following results:

$$
\frac{\partial N_{1}}{\partial X_{1}}>0, \frac{\partial N_{2}}{\partial X_{2}}<0, \frac{\partial N_{1}}{\partial X_{2}}>0, \frac{\partial N_{2}}{\partial X_{1}}<0 .
$$

Therefore, candidate 1's negative spending rises when $X_{1}$ increases and falls when $X_{2}$ decreases. Candidate 2's negative spending rises when $X_{2}$ decreases and falls when $X_{1}$ increases.

To use (22) to make a simpler statement, again suppose that $X_{1}=1-X_{2}$ and $E_{1}=E_{2}=E$, and let the symmetric equilibrium be the starting point. Then, suppose $X_{1}$ rises and $X_{2}$ rises as well, so that, starting from equal degrees of centrism, candidate 1 becomes relatively more centrist (with $X_{1}>1-X_{2}$ ). Using (23), the rise in $X_{1}$ causes $N_{1}$ to increase and $N_{2}$ to fall, while the rise in $X_{2}$ has the same effects, leading to $N_{1}>N_{2} \cdot{ }^{10}$ Summarizing yields

Proposition 2. In the vicinity of a symmetric equilibrium where stated positions are fixed, the more-centrist candidate has the higher level of negative campaign spending.

This result may at first seem counterintuitive because a relatively extreme stated position for the opponent might appear to reduce, rather than increase, the incentive for the more-centrist candidate to make her opponent's perceived position more extreme via negative spending. Conversely, a relatively more-centrist position for the opponent might appear to increase, rather than reduce, the incentive for the more-extreme candidate to make her opponent's perceived position more extreme via negative spending.

To resolve these puzzles, note that the candidate 1's utility gain from shifting her own position rightward via positive spending, which is proportional to $1-2 F\left(Z_{1}\right)$, is smaller the larger is $Z_{1}$ (and thus $X_{1}$ ), capturing the diminishing benefits of moving toward the median preferred position among voters. As a result, the loss from diverting funds from positive to negative spending is smaller the larger is $X_{1}$. Similarly, the utility gain from shifting the opponent's position away from the median via negative spending, which equals the opponent's loss from this shift, is proportional to $2 F\left(Z_{2}\right)-1$. This gain is larger the larger is $Z_{2}$ (and thus $X_{2}$ ). Therefore, candidate 1's negative spending is encouraged by larger values of both $X_{1}$ and $X_{2}$. 
Since the discussion surrounding (21) established that symmetric increases in the E's raise the symmetric $N$, it follows that the positive own budget effect in (22) is absolutely larger than the negative opponent's budget effect (when evaluated at a symmetric equilibrium). In addition, recalling that symmetric changes in centrism have no effect on the $N$ 's, it follows that the own $X$ effect in (23) is exactly cancelled by the opponent's $X$ effect. Therefore, even though a shift toward asymmetric degrees of centrism changes the $N$ 's, symmetric changes in the $X$ 's have no effect.

\subsection{Analysis with chosen $X$ 's: The $\lambda=1$ case}

The analysis now turns to the case where the $X$ 's as well as the $N$ 's are decision variables for the candidates. Given (2) and first-order conditions (14) and (16), the chosen policy positions satisfy

$$
X_{1}-B_{1}>0, \quad X_{2}-B_{2}<0
$$

Intuitively, candidate 1 chooses a policy position closer to the center than her ideology in order to increase the winning probability, taking account of the disutility of diverging from that ideology, and candidate 2 does the same. The set of equalities in (20) is expanded to include a fourth ratio, $\left(X_{1}-B_{1}\right) /\left(B_{2}-X_{2}\right)$.

When the $X^{\prime}$ 's are chosen, comparative-static analysis is unfortunately infeasible given the number and complexity of the equilibrium conditions, (14)-(17). However, partial results for the case where $\lambda=1$ can be established using an approach that does not rely on comparative statics. Even though comparative-static calculations are not used, the second-order conditions for the problem nevertheless must be satisfied. But the ambiguity of the sign of the Hessian determinant means that satisfaction is not guaranteed, even under symmetry or the previous sufficient condition. Therefore, the required positivity of the Hessian determinant is assumed.

The non-comparative-static approach relies on the following result:

Lemma. If $E_{1}=E_{2}=E$, so that campaign budgets are equal, and $\lambda=1$, then the equalities $N_{1}=P_{2}$ and $N_{2}=P_{1}$ hold.

To establish the lemma, assume that $\lambda=1$ and that, contrary to the claim, $N_{1}<P_{2}$ holds, 
which implies $N_{1}<E-N_{2}$ and hence that $N_{2}<E-N_{1}=P_{1}$. Then $h^{\prime}\left(N_{1}\right)>h^{\prime}\left(P_{2}\right)$ and $h^{\prime}\left(P_{1}\right)<h^{\prime}\left(N_{2}\right)$, so that $h^{\prime}\left(N_{1}\right) / h^{\prime}\left(P_{1}\right)>h^{\prime}\left(P_{2}\right) / h^{\prime}\left(N_{2}\right)$ holds, violating (20). The case where $N_{1}>P_{2}$ can be ruled out in parallel fashion. ${ }^{11}$

The lemma can be used to draw a connection between ideologies and stated positions:

Proposition 3. If $\lambda=1$ and the campaign budgets are equal, then the candidate with the more-centrist ideology chooses the more-centrist stated position.

This result is established by first noting from (10) that $Z_{1}=X_{1}$ and $1-Z_{2}=1-X_{2}$ hold when $\lambda=1$ because the equalities $N_{1}=P_{2}$ and $N_{2}=P_{1}$ are then satisfied by the lemma. Next, suppose that $B_{1}>1-B_{2}$ holds but that $X_{1} \leq 1-X_{2}$ also holds, contradicting the conclusion of the proposition. Then the first ratio in (20) exceeds 1, but the new fourth ratio, which can be written $\left(X_{1}-B_{1}\right) /\left[\left(1-X_{2}\right)-\left(1-B_{2}\right)\right]$, is less than 1 , a contradiction that establishes the first part of the proposition. The second part follows by the same argument.

Proposition 3 generates an additional conclusion analogous to Proposition 2:

Proposition 4. If $\lambda=1$ and the campaign budgets are equal, then the candidate with the more-centrist ideology has the higher level of negative campaign spending.

This result is established by noting that, since $Z_{1}=X_{1}>1-X_{2}=1-Z_{2}$ holds when $B_{1}>1-B_{2}$ by Proposition 3 and the lemma, the first ratio in (20) is less than one. As a result, $h^{\prime}\left(N_{1}\right) / h^{\prime}\left(E-N_{1}\right)<1$ and $h^{\prime}\left(E-N_{2}\right) / h^{\prime}\left(N_{2}\right)<1$ must hold, and these inequalities require $N_{1}>E / 2>N_{2} \cdot{ }^{12}$

It is interesting to note that, although this non-comparative-static approach allows a connection to be drawn between negative spending and ideologies, it cannot be used to establish an analog to Proposition 1, which relates negative spending to budgets, for the case where the $X^{\prime}$ 's are chosen. ${ }^{13}$

\section{Positive (Negative) Spending Shifts Valence (Position)}

\subsection{The model and first-order conditions}

Instead of following Harrington and Hess (1996) by assuming that positive and negative 
campaign spending both shift the perceived positions of the candidates, suppose that these expenditures play different roles. Specifically, suppose that a candidate's positive campaign spending is designed to shift her own valence in a favorable direction, while her negative spending is meant to shift her opponent's position toward a more-extreme location. With positive spending not affecting the Z's, (10) and (11) become

$$
Z_{1}=X_{1}-\lambda h\left(N_{2}\right), \quad Z_{2}=X_{2}+\lambda h\left(N_{1}\right)
$$

As for the effect of valence-shifting positive spending, such spending is assumed to shift the mean of the valence distribution from section 2. In particular, rather than assuming that the valence term $v_{x}$ is uniformly distributed over the interval $[-\beta, \beta]$, the support of the distribution is assumed to be $[-\beta+G, \beta+G]$, where $G$ captures the effect of positive campaigning (the density thus remains $1 / \beta$ ). $G$ depends on positive campaign spending levels via the relationship

$$
G=g\left(P_{1}\right)-g\left(P_{2}\right)
$$

where $g^{\prime}>0$ and $g^{\prime \prime}<0$. Thus, recalling that a large value of $v_{x}$ favors candidate 1 , an increase in 1's positive spending shifts the valence distribution in her favor (to the right) whereas an increase in candidate 2's spending shifts it in her favor (to the left). Note that $g$ is a different function from $h$ given the different targets of the spending (valence vs. position). ${ }^{14}$

To see the effect of this modification on the candidates' objective functions, note that the upper limit of integration in (4) is replaced by $\beta+G=\beta+g\left(P_{1}\right)-g\left(P_{2}\right)$. As a result, the expression on the right-hand side of the equation is replaced by $Z_{1}-Z_{2}+g\left(P_{1}\right)-g\left(P_{2}\right)+s$. Since the same replacement occurs in (4) and (5), $s$ is effectively replaced by $g\left(P_{1}\right)-g\left(P_{2}\right)+s$ wherever it appears. The integration leading to (9) proceeds as before, leading to the following expression for candidate 1's probability of winning the election:

$$
\frac{1}{2}+\frac{1}{2 \alpha}\left[Z_{1}+g\left(P_{1}\right)-Z_{2}-g\left(P_{2}\right)+2 \int_{Z_{1}}^{Z_{2}} F(x) d x\right] .
$$


Substituting for the Z's from (25), and subtracting the disutility of divergence from beliefs, candidate 1's utility function is written

$$
\begin{gathered}
U_{1}=R\left(\frac{1}{2}+\frac{1}{2 \alpha}\left[X_{1}-\lambda h\left(N_{2}\right)+g\left(P_{1}\right)-X_{2}-\lambda h\left(N_{1}\right)-g\left(P_{2}\right)+2 \int_{X_{1}-\lambda h\left(N_{2}\right)}^{X_{2}+\lambda h\left(N_{1}\right)} F(x) d x\right]\right) \\
-\gamma\left(X_{1}-B_{1}\right)^{2}
\end{gathered}
$$

Relative to the utility function (13) from the model of section 3, (28) differs in the absence of positive spending terms in the integral's limits of integration, a consequence of the assumption that positive spending does not affect the candidates' positions, altering only valence.

As before, candidate 2's probability of winning the election is one minus the expression in (27). As a result, her objective function is given by (28) with the first plus sign replaced by a minus sign, and with the last term replaced by $-\gamma\left(X_{2}-B_{2}\right)^{2}$.

Substituting $P_{i}=E_{i}-N_{i}$ in the two objection functions, $i=1$, 2, first-order conditions are computed, again assuming Nash behavior on the part of the candidates. Treating both $N_{i}$ and $X_{i}, i=1,2$, as decision variables, the first-order conditions for candidate 1 are

$$
\begin{aligned}
& U_{1}^{X_{1}} \simeq\left(1-2 F_{1}\right)-\mu\left(X_{1}-B_{1}\right)=0 \\
& U_{1}^{N_{1}} \simeq\left(2 F_{2}-1\right) \lambda h^{\prime}\left(N_{1}\right)-g^{\prime}\left(P_{1}\right)=0 .
\end{aligned}
$$

Note that (29) is the same as (14) but that (30) differs from (15) in the absence of the $\left(1-2 F_{1}\right)$ factor multiplying the positive spending derivative $g^{\prime}\left(P_{1}\right)$ (the $F$ arguments, of course, differ as well). Analogously, the first-order conditions for candidate 2 are

$$
\begin{aligned}
& U_{2}^{X_{2}} \simeq-\left(2 F_{2}-1\right)-\mu\left(X_{2}-B_{2}\right)=0 \\
& U_{2}^{N_{2}} \simeq\left(1-2 F_{1}\right) \lambda h^{\prime}\left(N_{2}\right)-g^{\prime}\left(P_{2}\right)=0 .
\end{aligned}
$$

The interpretation of these conditions is similar to that in the previous model. 
The second-order conditions for candidate 1 are (18) and

$$
U_{1}^{N_{1} N_{1}} \simeq 2 f_{2}\left(\lambda h_{1}^{\prime}\right)^{2}+\left(2 F_{2}-1\right) \lambda h_{1}^{\prime \prime}+g_{1}^{\prime \prime}<0
$$

where the 1 subscripts on $g^{\prime}$ and $h^{\prime}$ indicate evaluation at candidate 1 's values. As before, (33) contains both positive and negative terms, and its satisfaction must be assumed (unlike before, symmetry is of no help). As before, though, a condition ensuring satisfaction of (33) is $h^{\prime} / h \leq-h^{\prime \prime} / h^{\prime}$ (which holds if $h$ is a power function with exponent no larger than $1 / 2$ ). In

addition, since $U_{1}^{X_{1} N_{1}}$ is now zero, positivity of the Hessian determinant of $U_{1}$ is guaranteed if (33) holds.

\subsection{Basic condition}

Rearranging (29)-(32) yields the following condition in the fixed-X case:

$$
\frac{1-2 F_{1}}{2 F_{2}-1}=\frac{\lambda h^{\prime}\left(N_{1}\right)}{g^{\prime}\left(E_{1}-N_{1}\right)} \frac{g^{\prime}\left(E_{2}-N_{2}\right)}{\lambda h^{\prime}\left(N_{2}\right)} .
$$

Therefore, rather than being separately equal to the ratios of spending derivatives, the lefthand expression in (34) equals the product of these ratios in the modified model. In the symmetric case, with equal E's and symmetric $X$ 's, (34) is satisfied by a symmetric outcome, with $N_{1}=N_{2}$. However, asymmetric equilibria cannot be ruled out.

\subsection{Comparative-static analysis}

Comparative-static analysis with the $X$ 's fixed is simpler than in the previous model because the absence of $P_{2}$ from the $Z_{2}$ argument of $2 F_{2}-1$ in (30) means that the equation involves only $N_{1}$ and the exogenous quantities $X_{2}$ and $E_{1}$ (and similarly for (32)). Since $U_{1}^{N_{1} z}$ is positive for $z=X_{2}, E_{1}, \lambda$, the derivatives $\partial N_{1} / \partial z=-U_{1}^{N_{1} z} / U_{1}^{N_{1} N_{1}}$ are positive given (33), so that

$$
\frac{\partial N_{1}}{\partial X_{2}}, \quad \frac{\partial N_{1}}{\partial E_{1}}, \quad \frac{\partial N_{1}}{\partial \lambda}>0, \quad \frac{\partial N_{1}}{\partial X_{1}}=\frac{\partial N_{1}}{\partial E_{2}}=0
$$

with the effects on $N_{2}$ (from (32)) given by reversing the indexes. 
Although symmetry has not been required to sign these derivatives, suppose nevertheless that the starting point is a symmetric equilibrium and that $E_{1}$ increases and $E_{2}$ decreases. From (35), the first change raises $N_{1}$ while having no effect on $N_{2}$, while the second change reduces $N_{2}$ and has no effect on $N_{1}$. Thus, $N_{1}>N_{2}$ holds after the change, yielding an analog to Proposition 1 for the modified model. Similarly, consider the effect of asymmetric stated positions, starting at a symmetric equilibrium and then raising both $X_{1}$ and $X_{2}$. The second change raises $N_{1}$ from (35) while having no effect on $N_{2}$, while the first change reduces $N_{2}$ while having no effect on $N_{1}$, again yielding $N_{1}>N_{2}$ and establishing an analog to Proposition 2 for the modified model.

Note that, despite the similar conclusions, the outcomes in Propositions 1 and 2 and their analogs arise through different channels. While both the own and opponent's $E$ values affect a candidate's $N$ in the previous model, only the own effect is present here. Similarly, while both the own and opponent's $X$ values previously affected a candidate's $N$, only the opponent's $X$ effect is present here.

While a general comparative-static analysis of the case where both the $N$ 's and the $X$ 's are choice variables was unworkable in the previous model, it is feasible in the modified model. Although this analysis would normally require a complex total differentiation of the full equation system (29)-(32), a more streamlined approach is possible, as follows.

Using (30) and (31),

$$
-\frac{g^{\prime}\left(E_{1}-N_{1}\right)}{\lambda h^{\prime}\left(N_{1}\right)}=1-2 F_{2}=\mu\left(X_{2}-B_{2}\right)
$$

so that

$$
X_{2}=B_{2}-\frac{g^{\prime}\left(E_{1}-N_{1}\right)}{\mu \lambda h^{\prime}\left(N_{1}\right)} .
$$

Eliminating $X_{2}$ in (30) using (37), the equation can be rewritten as

$$
\Gamma=\left\{2 F\left[B_{2}-\frac{g^{\prime}\left(E_{1}-N_{1}\right)}{\mu \lambda h^{\prime}\left(N_{1}\right)}+\lambda h\left(N_{1}\right)\right]-1\right\} \lambda h^{\prime}\left(N_{1}\right)-g^{\prime}\left(E_{1}-N_{1}\right)=0
$$


a condition that determines $N_{1}$ as a function of parameters. Differentiating (38) with respect to $N_{1}$ yields

$$
\Gamma_{N_{1}}=U_{1}^{N_{1} N_{1}}+2 f_{2} \frac{g_{1}^{\prime \prime} h_{1}^{\prime}+g_{1}^{\prime} h_{1}^{\prime \prime}}{\mu \lambda\left(h_{1}^{\prime}\right)^{2}}<0
$$

using the second-order condition (33) (the negative second term captures the effect that operates through the new ratio expression in the argument of $F$ ).

Since it easily seen that $\Gamma_{E_{1}}, \Gamma_{B_{2}}, \Gamma_{\mu}, \Gamma_{\lambda}>0$ hold, ${ }^{15}$ while the effects of other parameters on $\Gamma$ are zero, the signs of the comparative-static derivatives $\partial N_{1} / \partial z=-\Gamma_{z} / \Gamma_{N_{1}}, z=E_{1}, B_{2}, \mu, \lambda$, are all positive:

$$
\frac{\partial N_{1}}{\partial E_{1}}>0, \frac{\partial N_{1}}{\partial B_{2}}>0, \frac{\partial N_{1}}{\partial \mu}>0, \frac{\partial N_{1}}{\partial \lambda}>0, \frac{\partial N_{1}}{\partial E_{2}}=\frac{\partial N_{1}}{\partial B_{1}}=0 .
$$

Note that the relevant parts of (40) match (35) with the $X$ 's replaced by $B$ 's. A new effect, however, operates through the parameter $\mu$, with (40) showing that an increase in $\mu$ raises negative spending. Recalling that $\mu=4 \alpha \gamma / R$, a higher $\mu$ can be caused by an increase in the disutility of divergence from ideology $(\gamma)$, a decrease in the benefits of holding office $(R)$, or an increase in the dispersion of the common electoral shock $(\alpha)$. The implication is that any of these changes leads to an increase in negative spending. ${ }^{16}$

Reversing the second inequality in (40), the negative-spending effects for candidate 2 are

$$
\frac{\partial N_{2}}{\partial E_{2}}>0, \frac{\partial N_{2}}{\partial B_{1}}<0, \frac{\partial N_{2}}{\partial \mu}>0, \frac{\partial N_{2}}{\partial \lambda}>0, \frac{\partial N_{2}}{\partial E_{1}}=\frac{\partial N_{2}}{\partial B_{2}}=0
$$

Using (37) and the expressions for the derivatives in (40), the appendix establishes ${ }^{17}$

$$
1>\frac{\partial X_{2}}{\partial B_{2}}>0, \frac{\partial X_{2}}{\partial \mu}>0
$$

Reversing the last sign in (42), the stated-position effects for candidate 1 are:

$$
1>\frac{\partial X_{1}}{\partial B_{1}}>0, \frac{\partial X_{1}}{\partial \mu}<0
$$


Therefore, stated positions become more extreme (moving closer to ideologies) as $\mu$ increases, a change that is especially transparent when the higher $\mu$ comes from a greater disutility of divergence from ideology $(\gamma)$. When ideologies change, stated positions shift in the same direction, but the shift is smaller.

These results can be used to draw conclusions about the effect of asymmetric conditions like those in Propositions 1, 2 and 4 by repeating the arguments following (35). However, these conclusions differ in that the $B$ 's are held fixed rather than the $X$ 's. Because a budget increase raises own negative spending while not affecting that of the opponent from (40) and (41), an analog to Proposition 2 follows:

Proposition 5. In the modified model with all choices endogenous, if ideologies are symmetric, then the candidate with the larger budget has the higher level of negative campaign spending.

Because (40) and (41) show that a more-centrist ideology for the opponent reduces own negative spending while own-ideology has no effect, an analog to Proposition 2 follows:

Proposition 6. In the modified model with all choices endogenous, if budgets are equal, then the candidate with the more-centrist ideology has the higher level of negative campaign spending.

The effect of symmetric changes in ideologies and budgets can be appraised, paralleling the discussion following (21) for the fixed- $X$ version of the first model. Starting at a symmetric equilibrium, common increases in the $E$ 's raise negative spending for both candidates given (40) and (41), matching the earlier conclusion. However, while negative spending was independent of positions in the symmetric fixed- $X$ version of the first model, a different conclusion applies for the modified model. Starting at a symmetric equilibrium, a common movement of the $B$ 's toward the center reduces negative spending for both candidates, as can be seen from (40) and (41).

\subsection{Summary}

The paper's results are summarized in Table 1, which shows the effects of the parameters on negative campaign spending for both versions of the model. Budget effects are mostly as 
expected, although the negative effect of the opponent's budget in the fixed- $X$ version of the first model is not predictable. The positive impacts of the productivity of negative spending are also natural, although the positive effect of $\mu$ in the modified model is again unpredictable.

The most important results of the paper concern the effects of relative centrism on negative campaign spending. Although the table entries for centrism's effects are varied, they all show that relative centrism raises a candidate's negative spending compared to her opponent's. This effect arises through the reverse effects of own and opponent centrism in the fixed- $X$ version of the first model, as seen in the first column of Table 1 . When $X$ is chosen in that model, comparative-static results are unavailable but Proposition 4 establishes the same conclusion in a different fashion, as seen in the second column. In the modified model, the relativecentrism effect arises entirely through the opponent, with greater centrism for the opponent (which reduces relative own-centrism) lowering own negative spending, as seen in the last two columns of the table.

\section{Conclusion}

This paper has extended the small existing theoretical literature on negative campaigning, building on work by Harrington and Hess (1996). While their analysis explores the determinants of negative campaign spending using a classic spatial voting model, this paper relies instead on a probabilistic voting model, extending the use of this popular model to a new setting. By using two different versions of the model based on different assumptions on the targeting of campaign spending, the paper generates a host of results. However, the main lesson, which emerges in fairly robust fashion regardless of specific assumptions, is that negative campaign spending is higher for the relatively more-centrist candidate. The more-extreme candidate in the electoral contest devotes, by contrast, comparatively more of her funds to positive campaign spending. This result, which at first appears unrelated to the main findings of Harrington and Hess (1996) and Chakrabarti's (2007) extension of their model, is actually consistent with their results upon deeper examination. Therefore, spatial models of negative campaigning can generate a unified set of conclusions despite fundamental differences in their structures. 
As mentioned in the introduction, the analysis generates a prediction that has not been tested in the empirical literature on the determinants of negative campaign spending. A test could recognize that a Democrat competing with a Republican opponent in a Democraticleaning (Republican-leaning) electoral district is the relatively more-centrist (less-centrist) candidate. This Democratic candidate is predicted by the model to spend more (less) on negative campaigning than her opponent, other things equal. The test could then add a dummy variable to the negative-spending regression that captures whether the particular candidate's party affiliation matches the leaning of the district. If the model's prediction is correct, this variable's coefficient should be positive. 


\section{Appendix}

\section{Proof of Proposition 1}

The relevant derivatives of the expressions in the first-order conditions (15) and (17) are given by

$$
\begin{aligned}
& U_{1}^{N_{1} N_{1}} \simeq 2 f_{2}\left(\lambda h^{\prime}\left(N_{1}\right)\right)^{2}+\left[2 F_{2}-1\right] \lambda h^{\prime \prime}\left(N_{1}\right)-2 f_{1}\left(h^{\prime}\left(P_{1}\right)\right)^{2}+\left[1-2 F_{1}\right] h^{\prime \prime}\left(P_{1}\right) \\
& U_{2}^{N_{2} N_{2}} \simeq-2 f_{2}\left(h^{\prime}\left(P_{2}\right)\right)^{2}+\left[2 F_{2}-1\right] h^{\prime \prime}\left(P_{2}\right)+2 f_{1}\left(\lambda h^{\prime}\left(N_{2}\right)\right)^{2}+\left[1-2 F_{1}\right] \lambda h^{\prime \prime}\left(N_{2}\right) \\
& U_{1}^{N_{1} N_{2}} \simeq 2 f_{2} h^{\prime}\left(P_{2}\right) \lambda h^{\prime}\left(N_{1}\right)-2 f_{1} h^{\prime}\left(P_{1}\right) \lambda h^{\prime}\left(N_{2}\right) \\
& U_{2}^{N_{2} N_{1}} \simeq-2 f_{2} h^{\prime}\left(P_{2}\right) \lambda h^{\prime}\left(N_{1}\right)+2 f_{1} h^{\prime}\left(P_{1}\right) \lambda h^{\prime}\left(N_{2}\right) \\
& U_{1}^{N_{1} E_{1}} \simeq 2 f_{1}\left(h^{\prime}\left(P_{1}\right)\right)^{2}-\left[1-2 F_{1}\right] h^{\prime \prime}\left(P_{1}\right) \\
& U_{1}^{N_{1} X_{1}} \simeq 2 f_{1} h^{\prime}\left(P_{1}\right) \\
& U_{2}^{N_{2} E_{1}} \simeq-2 f_{1} h^{\prime}\left(P_{1}\right) \lambda h^{\prime}\left(N_{2}\right) \\
& U_{2}^{N_{2} X_{1}} \simeq-2 f_{1} \lambda h^{\prime}\left(N_{2}\right) .
\end{aligned}
$$

In using Cramer's rule to compute comparative-static derivatives, the sign of the determinant $H \equiv U_{1}^{N_{1} N_{1}} U_{2}^{N_{2} N_{2}}-U_{1}^{N_{1} N_{2}} U_{2}^{N_{2} N_{1}}$ is needed. Since $U_{1}^{N_{1} N_{1}}$ and $U_{2}^{N_{2} N_{2}}$ are negative at the

symmetric equilibrium and since $U_{1}^{N_{1} N_{2}}$ and $U_{2}^{N_{2} N_{1}}$ are both zero under symmetry (see above), $H>0$ holds. Note that if the sufficient condition discussed in the text holds, so that the second derivatives are always negative, then $H>0$ is also satisfied given that the cross partial derivatives have opposite signs away from a symmetric equilibrium, leading to full satisfaction of the second-order conditions.

$$
\begin{aligned}
& \text { Using } H>0 \\
& \frac{\partial N_{1}}{\partial E_{1}}=\frac{1}{H}\left\{U_{1}^{N_{1} N_{2}} U_{2}^{N_{2} E_{1}}-U_{2}^{N_{2} N_{2}} U_{1}^{N_{1} E_{1}}\right\} \\
& =\frac{1}{H}\left\{\left[1-2 F_{1}\right] h^{\prime \prime}\left(P_{1}\right) U_{2}^{N_{2} N_{2}}-2 f_{1}\left(h^{\prime}\left(P_{1}\right)\right)^{2}\left[\left(2 F_{2}-1\right) h^{\prime \prime}\left(P_{2}\right)+\left(1-2 F_{1}\right) \lambda h^{\prime \prime}\left(N_{2}\right)\right]\right\}>0 .
\end{aligned}
$$


The second equality uses the derivative expressions above along with (20). The inequality in (a1) follows because $U_{2}^{N_{2} N_{2}}<0$ holds and because $2 F_{2}-1>0$ and $1-2 F_{1}>0$.

Analogously,

$$
\begin{array}{rlr}
\frac{\partial N_{2}}{\partial E_{1}} & =\frac{1}{H}\left\{U_{2}^{N_{2} N_{1}} U_{1}^{N_{1} E_{1}}-U_{1}^{N_{1} N_{1}} U_{2}^{N_{2} E_{1}}\right\} & \text { (a2) } \\
& =\frac{2}{H}\left\{\left[1-2 F_{1}\right] f_{2} \lambda h^{\prime}\left(N_{1}\right) h^{\prime}\left(P_{2}\right) h^{\prime \prime}\left(P_{2}\right)+\left[2 F_{2}-1\right] f_{1} h^{\prime}\left(P_{1}\right) \lambda h^{\prime}\left(N_{2}\right) \lambda h^{\prime \prime}\left(N_{1}\right)\right\}< & <.
\end{array}
$$

By switching the candidate index and budget indices, it is easy to verify that

$$
\frac{\partial N_{1}}{\partial E_{2}}<0, \frac{\partial N_{2}}{\partial E_{2}}>0
$$

Proof of Proposition 2

$$
\begin{aligned}
\frac{\partial N_{1}}{\partial X_{1}} & =\frac{1}{H}\left\{U_{1}^{N_{1} N_{2}} U_{2}^{N_{2} X_{1}}-U_{2}^{N_{2} N_{2}} U_{1}^{N_{1} X_{1}}\right\} \\
& =-\frac{2 f_{1} h^{\prime}\left(P_{1}\right)}{H}\left\{\left[1-2 F_{1}\right] \lambda h^{\prime \prime}\left(N_{2}\right)+\left[2 F_{2}-1\right] h^{\prime \prime}\left(P_{2}\right)\right\}>0
\end{aligned}
$$

and

$$
\begin{aligned}
\frac{\partial N_{2}}{\partial X_{1}} & =\frac{1}{H}\left\{U_{2}^{N_{2} N_{1}} U_{1}^{N_{1} X_{1}}-U_{1}^{N_{1} N_{1}} U_{2}^{N_{2} X_{1}}\right\} \\
& \left.=\frac{2 f_{1} \lambda h^{\prime}\left(N_{2}\right)}{H}\left\{\left[1-2 F_{1}\right] h^{\prime \prime}\left(P_{1}\right)+\left[2 F_{2}-1\right] \lambda h^{\prime \prime}\left(N_{1}\right)\right)\right\}<0
\end{aligned}
$$

By switching the candidate and budget indices, it is easy to verify that

$$
\frac{\partial N_{1}}{\partial X_{2}}>0, \quad \frac{\partial N_{2}}{\partial X_{2}}<0
$$


Derivation of (42)

Differentiating (37) with respect to $B_{2}$ yields

$$
\frac{\partial X_{2}}{\partial B_{2}}=1+\Omega \frac{\partial N_{1}}{\partial B_{2}}
$$

where

$$
\Omega=\frac{g_{1}^{\prime \prime} h_{1}^{\prime}+g_{1}^{\prime} h_{1}^{\prime \prime}}{\mu \lambda\left(h_{1}^{\prime}\right)^{2}}<0 .
$$

Substituting for $\partial N_{1} / \partial B_{2},(a 1)$ reduces to

$$
\frac{\partial X_{2}}{\partial B_{2}}=1-\frac{f_{2} \Omega}{U_{1}^{N_{1} N_{1}}+2 f_{2} \Omega}>0 .
$$

Note that (a9), while positive, is also less than one. Differentiating (37) with respect to $\mu$ yields

$$
\begin{aligned}
\frac{\partial X_{2}}{\partial \mu} & =\frac{g_{1}^{\prime}}{\mu^{2} \lambda h_{1}^{\prime}}+\Omega \frac{\partial N_{1}}{\partial \mu} \\
& =\frac{g_{1}^{\prime}}{\mu^{2} \lambda h_{1}^{\prime}}\left(1-\frac{2 f_{2} \Omega}{U_{1}^{N_{1} N_{1}}+2 f_{2} \Omega}\right)>0 .
\end{aligned}
$$


Table 1

Parameter Impacts on Negative Spending

\begin{tabular}{|c|c|c|}
\hline All Spendi & ects Position & $\begin{array}{l}\text { Negative Spending Affects Position } \\
\text { Positive Spending Affects Valence }\end{array}$ \\
\hline$X$ 's Fixed & $X$ 's Chosen & $X^{\prime}$ s Chosen \\
\hline
\end{tabular}

BUDGET

- own

- opponent's

$+\quad$ ?

$+\quad+$

$0 \quad 0$

\section{CENTRISM}

\begin{tabular}{lcccc}
- own & + & $\begin{array}{l}\text { higher for more- } \\
\text { centrist candidate } \\
\text { if } \lambda=1\end{array}$ & 0 & 0 \\
- opponent's & - & $?$ & + & + \\
\hline$\lambda$ & + & $?$ & n.a. \\
\hline$\mu$ & n.a. & & \\
\hline \hline
\end{tabular}




\section{References}

Ansolbehere, S., Iynegar, S., Simon, A., Valentino, N., 1994. Does attack advertising demobilize the electorate? American Political Science Review 88, 829-838.

Chakrabarti, S., 2007. A note on negative electoral advertising: Denigrating character vs. portraying extremism. Scottish Journal of Political Economy 54, 136-149.

Cotton, C., 2009. Should we tax or cap political contributions? A lobbying model with policy favors and access. Journal of Public Economics 93, 831-842.

Cox, G., Munger, M., 1989. Closeness, expenditures and turnout in the 1982 US house elections. American Political Science Review 83, 217-230.

Damore, D.F., 2002. Candidate strategy and the decision to go negative. Political Research Quarterly 55, 669-685.

Downs, A., 1957. An Economic Theory of Democracy. Harper \& Row: New York.

Endersby, J., Galatas, S., Rackaway, C., 2002. Closeness counts in Canada: Voter participation in the 1993 and 1997 federal elections. Journal of Politics 64, 610-631.

Enelow, J., Hinich, M., 1989. A general probabilistic spatial theory of election. Public Choice 61, 101-113.

Erickson, R., Palfrey, T., 2000. Equilibria in campaign spending games: Theory and data. American Political Science Review 94, 595-609.

Franz, M., Ridout, T., 2007. Does political advertising persuade? Political Behavior 29, 465-491.

GERBER, A., 1998. Estimating the effect of campaign spending on senate election outcomes using instrumental variables. American Political Science Review 92, 401-411.

Grossman, G., Helpman, E., 1996. Electoral competition and special interest politics. Review of Economics Studies 63, 265-286.

Harrington, J., JR., Hess, G., 1996. A spatial theory of positive and negative campaigning. Games and Economic Behavior 17, 209-229.

Haynes, A.A., Rhine, S.L., 1998. Attack politics in presidential nomination campaigns: An examination of the determinants of intermediated negative messages against opponents. 
Political Research Quarterly 51, 691-721.

Herrera, H., Levine, D., Martinelli, C., 2008. Policy platforms, campaign spending and voter participation. Journal of Public Economics 92, 501-513.

Hotelling, H., 1929. Stability in competition, Economic Journal 39, 41-57.

Kahn, K.F., 1993. Gender differences in campaign messages: The political advertisements of men and women candidates for the U.S. Senate. Political Research Quarterly 46, 481-502.

KonRAD, K., 2004. Inverse campaigning. Economic Journal 114, 69-82.

Lau, R.R., Pomper G., 2001. Negative campaiging by U.S. Senate candidates. Party Politics 7, 69-87.

Lau, R.R., Rovner, I.B., 2009. Negative campaigning. Annual Review of Political Science 12, 285-306.

LEVITT, S., 1994. Using repeat challengers to estimate the effect of campaign spending on election outcomes in the US House. Journal of Political Economy 102, 777-798.

LindBeCK, A., Weibull, J., 1987. Balanced-budget redistribution as the outcome of political competition. Public Choice 52, 273297.

Milligan, K., Rekkas, M., 2008. Campaign spending limits, incumbent spending, and election outcomes. Canadian Journal of Economics 41, 1351-1374.

Pastine, I., Pastine, T., 2012. Incumbency advantage and political campaign spending limits. Journal of Public Economics 96, 20-32.

Persson, T., Tabellini, G., 2000. Political Economics. The MIT Press: Cambridge.

Peterson, D.A.M., Duupe, P.A., 2005. When primary campaigns go negative: The dterminants of campaign negativity. Political Research Quarterly 58, 45-54.

Polborn, M., Yi, D., 2006. Informative positive and negative campaigning. Quarterly Journal of Political Science 1, 351-371.

Proctor, D., Schenck-Hamlin, W., Hasse, K., 1994. Exploring the role of gender in the development of negative political advertisements. Women and Politics 14, 1-22.

Sahuguet, N., Persico, N., 2006. Campaign spending regulation in a model of redistributive politics. Economic Theory 28, 95-124. 
Skaperdas, S., Grofman, B., 1995. Modeling negative campaigning. American Political Science Review 89, 49-61.

Wattenberg, M.P., Brians, C.L., 1999. Negative campaign advertising: Demobilizer or mobilizer? American Political Science Review 93, 891-899. 


\section{Footnotes}

${ }^{*}$ We thank Ami Glazer for helpful commments, although any shortcomings in the paper are our responsibility.

${ }^{1}$ Levitt (1994), Gerber (1998), Erickson and Palfrey (2000), and Franz and Ridout (2007) study the effects of campaign spending on election outcomes. Cox and Munger (1989), Endersby et al. (2002), and Herrera et al. (2008) consider the effects of campaign spending on voter participation. Sahuguet and Persico (2006), Milligan and Rekkas (2008), Cotton (2009), and Pastine and Pastine (2012) analyze the effect of campaign spending limits.

${ }^{2}$ Voters are designated as males and candidates as females.

${ }^{3}$ Konrad (2004) develops a model of "inverse campaigning," in which a candidate informs voters that a small subset of individuals will benefit from an opponent's proposal, which the candidate opposes. This information may shift benefit perceptions among the remaining voters, increasing overall support for the candidate.

${ }^{4}$ Integrating by parts, the last term becomes

$$
\int_{Z_{1}}^{Z_{2}} \frac{x}{\beta} f(x) d x=\frac{1}{\beta}[x F(x)]_{Z_{1}}^{Z_{2}}-\int_{Z_{1}}^{Z_{2}} \frac{1}{\beta} F(x) d x=\frac{1}{\beta}\left[Z_{2} F\left(Z_{2}\right)-Z_{1} F\left(Z_{1}\right)-\int_{Z_{1}}^{Z_{2}} F(x) d x\right] .
$$

${ }^{5}$ It is also assumed that the limit of $h^{\prime}$ as its argument approaches zero is infinity, an Inada condition that ensures interior solutions.

${ }^{6}$ For example, during the 2012 U.S. presidential-election campaign, the Obama campaign portrayed Mr. Romney as an extremely conservative candidate, exemplified by the $47 \%$ remark. Such a portrayal is viewed as negative spending in the sense that it makes the conservative candidate look more conservative. At the same time, the Obama campaign emphasized that Mr. Obama has a strong record of national security, exemplified by the assasination of Osama Bin Laden, and such emphasis is viewed as positive spending that moves the liberal candidate toward the center.

${ }^{7}$ This condition can be shown to ensure that the sum of the first two terms in (19) is zero or negative. Satisfaction of the condition ensures that the equilibrium is unique and stable.

${ }^{8} \partial N / \partial \lambda=-h^{\prime}(N) h^{\prime}(E-N) / \lambda \Omega>0$ and $\partial N / \partial E=h^{\prime}(N) h^{\prime \prime}(E-N) / \Omega>0$, where $\Omega=$ $h^{\prime \prime}(N) h^{\prime}(E-N)+h^{\prime}(N) h^{\prime \prime}(E-N)<0$. 
${ }^{9}$ Note that this outcome could also result from a rise in $E_{1}$ hold $E_{2}$ fixed or a drop in $E_{2}$ holding $E_{1}$ fixed.

${ }^{10}$ Note that this outcome could also result from a rise in $X_{1}$ holding $X_{2}$ fixed or a rise in $X_{2}$ holding $X_{1}$ fixed.

${ }^{11}$ The lemma is a special case of a more general statement: if $E_{1}=E_{2}$, then $N_{1}>(=)<P_{2}$ and $N_{2}>(=)<P_{1}$ hold as $\lambda>(=)<1$. This statement can be established by adapting the above argument.

${ }^{12}$ Note that, when $\lambda=1$ this argument can also be used to establish Proposition 2 without reliance on comparative statics.

${ }^{13}$ The key result of Harrington and Hess (1996), that the candidate with the lower valence spends more on negative campaigning, is consistent with the results in Propositions 2 through 4 in this paper, once lower valence is translated into a more-centrist policy position, as noted in the Introduction. However, HH's result hinges on the strict concavity of the function capturing disutility from divergence between the voter's ideology and the candidate's position. By contrast, the results in this paper assume that voters' utility is linear, relying on the idea that a candidate's negative spending enables the candidate to capture a share of swing voters. Thus, HH rely on the second-order properties of preferences while the present analysis relies on first-order effects.

${ }^{14}$ Chakrabarti (2007) takes a similar approach but views $P$ as negative spending that reduces the opponent's relative valence, so that the RHS of (26) is reversed.

${ }^{15}$ Differentiation of $(38)$ shows that $\Gamma_{E_{1}}=-\left(2 f_{2} / \mu \lambda h_{1}^{\prime}+1\right) g_{1}^{\prime \prime}>0, \Gamma_{B_{2}}=f_{2}>0, \Gamma_{\mu}=$ $2 f_{2} g_{1}^{\prime} / \mu^{2} \lambda h_{1}^{\prime}>0$ and $\Gamma_{\lambda}=\left(2 F_{2}-1\right) \lambda h_{1}^{\prime}+2 f_{2}\left(h_{1}+g_{1}^{\prime} / \mu \lambda\right)>0$.

${ }^{16}$ In response to parameter changes, positive spending $P_{1}$ moves in a direction opposite to that of $N_{1}$, although in the $E_{1}$ case, the effect is ambiguous as before.

${ }^{17}$ The direction of $E_{1}$ 's impact on $X_{2}$ is ambiguous, as is the effect of $\lambda$. 